\title{
Timing of Susceptibility to Fusarium Head Blight in Winter Wheat
}

\author{
Christina Cowger, ${ }^{1, \dagger}$ Giovanni Beccari, ${ }^{2}$ and Yanhong Dong ${ }^{3}$ \\ ${ }^{1}$ USDA-ARS, Department of Entomology \& Plant Pathology, North Carolina State University, Raleigh, NC 27695, U.S.A. \\ ${ }^{2}$ Department of Agricultural, Food and Environmental Sciences, University of Perugia, 06123 Perugia, Italy \\ ${ }^{3}$ Department of Plant Pathology, University of Minnesota, St. Paul, MN 55108, U.S.A.
}

\begin{abstract}
The duration of wheat susceptibility to Fusarium infection has implications for risk forecasting, fungicide timing, and the likelihood that visible kernel damage may underpredict deoxynivalenol (DON) contamination. A field experiment was conducted to explore the impact of varying infection timings on Fusarium head blight (FHB) development in winter wheat. Trials in four successive years (2010 to 2013) in North Carolina utilized one susceptible and one moderately resistant cultivar possessing similar maturity, stature, and grain quality. Inoculum was applied in the form of sprayed Fusarium graminearum conidia. In the first year, the nine infection timings were from 0 to 21 days after anthesis (daa),

damage and kernel infection, and fungal spread as assayed via qPCR, the moderately resistant cultivar had at least a 2- to 3-day shorter window of susceptibility to damaging FHB infection than the susceptible cultivar. The results suggest that duration of susceptibility is an important aspect of cultivar resistance to FHB. In 2012, the window of susceptibility for both cultivars was extended by cold snaps during anthesis. After debranning in one year, the majority of DON was found to be in the bran fraction of kernels; there was also a trend for later infections to lead to a higher percentage of DON in the nonbran fraction, as well as a higher ratio of DON to FDK
\end{abstract} whereas in the following 3 years, they ranged from 0 to 13 daa. Infection progression was compared among inoculation timings by sampling spikes five to six times during grain-fill. Based on DON, percent kernel
Keywords: cereals and grains, cultivar/resistance, Fusarium graminearum, Fusarium head blight, infection timing, fungi, disease management
Fusarium head blight (FHB, or scab) of wheat (Triticum spp.) and barley (Hordeum vulgare) is a much-studied disease that lowers grain yield and contaminates grain with mycotoxins such as deoxynivalenol (DON). In the United States, the disease is mainly caused by Fusarium graminearum Schwabe sensu lato (teleomorph Gibberella zeae), a fungal pathogen with a wide host range, which produces DON and in some cases nivalenol. A patchy distribution of Fusarium strains producing "emerging mycotoxins" rather than DON has been detected in North Carolina (Cowger et al. 2020). The U.S. Food and Drug Administration (FDA) has set $1 \mu \mathrm{g} / \mathrm{g}$ as the advisory ceiling for DON in finished wheat products such as flour and bran that are intended for the human food supply (U.S. FDA 2010). U.S. flour mills often set a DON ceiling of $2 \mu \mathrm{g} / \mathrm{g}$ based on the experience that the DON concentration in the debranned flour will be $\leq 1 \mu \mathrm{g} / \mathrm{g}$ (Pate et al. 2003). The European Union has established maximum levels of DON in raw bread wheat and raw durum wheat of 1,250 and $1,750 \mu \mathrm{g} / \mathrm{kg}$ (EU 2006, 2007).

In wheat, extruded wheat anthers are the primary infection site (Parry et al. 1995). Anther infections often result in lack of kernel development, whereas slightly later infections lead to shriveled DONcontaining kernels (Fusarium-damaged kernels, or FDK), and still later infections can result in apparently sound kernels that nonetheless are

${ }^{\dagger}$ Corresponding author: C. Cowger; Christina.Cowger@usda.gov

Funding: This research was supported by SexSeed - Sexual Plant Reproduction - Seed Formation (H2020-MSCA-RISE-2015 Proposal ID 690946), the U.S. Wheat \& Barley Scab Initiative, and the U.S. Department of Agriculture.

The mention of company names or trade products does not imply that they are endorsed or recommended by the U.S. Department of Agriculture over other companies or similar products not mentioned. USDA is an equal opportunity provider and employer.

The author(s) declare no conflict of interest.

Accepted for publication 4 May 2020.

This article is in the public domain and not copyrightable. It may be freely reprinted with customary crediting of the source. The American Phytopathological Society, 2020. infected and contain mycotoxins (Cowger and Arellano 2010; Del Ponte et al. 2007; Hart et al. 1984; Schmale and Bergstrom 2003). Postanthesis moisture tends to increase DON and FDK (Cowger et al. 2009).

Previous studies have tried to discern when susceptibility to FHB infection declines in wheat, both in the field and under controlled conditions. For example, winter wheat was estimated to remain vulnerable to FHB and mycotoxin development up to 10 days after midflowering (Zadoks growth stage = GS 65) in the field (Cowger and Arellano 2010). In the greenhouse, inoculations of a susceptible winter wheat genotype with $F$. graminearum at 3 and 6 days after early anthesis ( 3 and 6 days after Zadoks GS 63 to 64; days after anthesis = daa) produced greater amounts of fungal biomass and secondary metabolites than those at 0 or 9 daa (Beccari et al. 2019).

Cowger and Arellano (2010) investigated the relationship between FDK and DON to try to understand reports of occasional occurrence of plump, healthy-looking grain with higher DON than would normally be expected. They found that the percentage of wheat samples with higher DON levels than visible kernel damage would predict was elevated under conditions that give rise to late infections and are marginal for disease. They predicted that periods of rain soon after anthesis might favor the scenario of low symptoms and/or low FDK coupled with problematic DON levels.

In addition, Cowger and Arellano (2013) investigated the progress of FHB development during grain-fill after inoculation at midanthesis. They found that DON levels declined in grain from medium milk (Zadoks GS 75, 15 daa) to harvest-ripeness (Zadoks GS 91 to 92, 55 daa) but increased in rachises and glumes until near harvest. FDK and percentage of infected kernels (PIK) increased from medium-milk until harvest-ripe stages and were elevated by postanthesis moisture compared with unirrigated treatments, suggesting that moist conditions during grain-fill can facilitate fungal spread within spikes by alleviating the effects of increasing water shortage as tissues senesce. Cowger and Arellano (2013) did not examine the effect of varying infection timings on these dynamics of FHB progress during grain-fill.

One of the challenges in precisely characterizing when infection may occur is that the window of anthesis may be narrower or wider depending on host genetics or environmental factors such as temperature and moisture. A detailed, specific understanding of the transition from vulnerability to reduced vulnerability would help us 
more accurately forecast the severity of FHB epidemics and could allow us to manipulate both host and environmental factors to better manage FHB. For risk forecasting, it would be helpful to better understand whether the transition from susceptibility to resistance occurs at a different time in susceptible cultivars versus more resistant cultivars. Knowledge of the factors that extend or shorten the window of susceptibility may shed light on issues such as why fungicide applications vary in effectiveness.

A multiyear field experiment was conducted with winter wheat, with the goal of more precisely characterizing the window of susceptibility to FHB. The experiment sought to identify how long wheat remains susceptible after anthesis and how quickly susceptibility declines. Additional objectives were to determine how infection timing affects the progression of fungal infection and DON during grain-fill.

\section{Materials and Methods}

Field experiment location and design. The experiment was conducted in the winter wheat crop years of 2009-2010, 2010-2011, 2011-2012, and 2012-2013 (hereafter referred to as 2010, 2011, 2012, and 2013). All experiments were planted at the Lake Wheeler Research Station near Raleigh, North Carolina. Conventional tillage with burial of crop residues was conducted before planting.

The experiment had a split-plot design with cultivar as the main plot and inoculation timing as the subplot. There were four replicate blocks; however, in 2010 only three replicate blocks were utilized due to problems with inoculation in the fourth block, and in 2012 one replicate of P26R12 was omitted from all analyses due to errors. Plots consisted of four adjacent 3.7-m rows, with the two outside rows serving as buffers and only the inner rows used for sampling purposes. Planting was accomplished with a Hege head-row planter (Wintersteiger, Salt Lake City, UT) on 22 October 2009, 19 October 2010, 25 October 2011, and 18 October 2012.

Two soft red winter wheat cultivars were planted each year. They were Pioneer 26R12 (P26R12; PI 631475), susceptible to FHB, and NC-Neuse (PI 633037), moderately resistant to FHB. These two cultivars were chosen because they were similar in other relevant respects: relatively tall stature, almost identical (late) maturity, and genetically high test weight.

The subplot treatments were nine different inoculation timings (described below) and an unsprayed control, or 10 treatments altogether. Thus, in each year there were a total of 80 plots (two cultivars $\times 10$ inoculation treatments $\times$ four replicates). A strip of rye (Secale cereale $\mathrm{L}$.) was planted around the experiment to enhance humidity.

Inoculation and irrigation. In the first year, inoculations were conducted at $0,7,9,11,13,15,17,19$, and 21 daa (the date when $50 \%$ of spikes were at $50 \%$ flowering). The results from that year indicated the need to more intensively investigate anthesis period itself, and so in the other three years, inoculations were conducted at $0,3,5$, $6,7,8,9,11$, and 13 daa. Each year, macroconidia of three $F$. graminearum isolates that had been derived from wheat spikes in North Carolina were produced by fermentation in mung tea and combined in equiproportional amounts. Conidia were centrifuged gently, resuspended in distilled water, counted by hemocytometer, and frozen in batches until use. Inoculation was conducted with a 19-liter backpack sprayer (Solo 435, Solo, Newport News, VA), and the center two rows of each plot were sprayed with $100 \mathrm{ml}$ of water containing $5 \times 10^{5} \mathrm{~F}$. graminearum macroconidia per milliliter and a drop of the surfactant Tween 20.

The 0 daa dates were 23 April (P26R12) or 25 April (NC-Neuse) 2010, 22 April (P26R12) or 24 April (NC-Neuse) 2011, 10 April 2012 (both cultivars), and 25 April (P26R12) or 27 April (NCNeuse) 2013. Starting at 0 daa each year, mist irrigation was supplied for $3 \mathrm{~h}$ in the morning (approximately 8:00 to 11:00 a.m.) and $3 \mathrm{~h}$ in the afternoon (approximately 2:00 to 5:00 p.m.). Mist irrigation continued until 28 daa. In 2012, due to error, irrigation did not commence immediately after the 0 -daa inoculation.

Disease assessment. Disease incidence was evaluated as the percentage of spikes with FHB symptoms, and disease severity as the average percentage of blighted spikelets on symptomatic spikes. Disease index was calculated as the product of incidence and severity divided by 100 and represented the percentage of visibly blighted spikelets in the plot. Disease was assessed shortly before spikes began to turn color and senesce, at soft dough stage (Zadoks GS 85), on 16 May 2010, 21 to 22 May 2011, 7 to 8 May 2012, and 20 May 2013. The assessment was conducted by blindly selecting five sets of 10 spikes each per plot and recording the percentage of blighted spikelets on each spike.

Sampling. Three sets of spike samples were collected. The first set, termed the "dai" set, was collected to follow the progress of infection from anthesis to maturity, and it consisted of spikes sampled at $14,21,28,35$, or 42 days after inoculation (dai) (also 49 dai in 2012). On each of those dates, 20 spikes were selected blindly from each plot and bulked. The second, "harvest-ripe" set consisted of 20 spikes blindly selected from each plot as a bulk at physiological maturity. These harvest-ripe samples were collected on the same day from every plot in 2010 and 2013 (10 June 2010 and 17 June 2013). In 2012, the "harvest-ripe" samples were those collected at 49 dai from the 0-, 3-, 5-, and 6-daa treatments and at 42 dai for the 7-, 8-, 9-, 11-, and 13-daa treatments, such that all samples were collected between 29 May and 4 June.

The third set of samples were for dissection to determine growth stage; these were collected in the first 3 years only. On each inoculation date and until Zadoks GS 92 (kernel hard), three spikes were collected per control plot and refrigerated until examination. Growth stage was determined by dissecting three spikelets from each spike to obtain an average growth stage.

Kernel damage and DON assays. To determine the percentage of FDK, 100 kernels were selected at random from each dai and harvestripe sample and assayed visually. The kernels were visually inspected and scored for gray-white and/or pink discoloration and mycelial growth. In each year, all estimates were made by the same assessor. As an additional measure of kernel damage, weights of randomly collected spikes were determined from each harvest-ripe sample in the first 3 years (30 spikes each in 2010 and 2011, 15 spikes in 2012).

The PIK was assayed in 2010 and 2012. For this assay, 100 kernels were selected at random from each dai and harvest-ripe sample. After surface sterilization in 5\% bleach for $2 \mathrm{~min}$, the kernels were plated on quarter-strength potato dextrose agar and incubated for 7 days at room temperature. Fusarium infection was determined by colony color and morphology.

From each dai and harvest-ripe sample, approximately $4 \mathrm{~g}$ of grain was analyzed for DON using gas chromatography-mass spectrometry.

Bran and nonbran DON. In debranned wheat, DON may be reduced by 15 to $78 \%$ (reviewed in Cheli et al. 2013). We hypothesized that the relative levels of infection in the pericarp and the endosperm of wheat kernels would vary depending on timing of infection. In 2012, to separate bran and nonbran fractions, samples were debranned in a Satake TM05 Testing Mill (Satake, Penrith, NSW, Australia).

In order to obtain large enough ( $\geq 15 \mathrm{~g}$ ) samples for successful debranning, later-collected samples (in the 21- to 49-dai range) were chosen because of their greater mass, and samples were combined across two collection timings; that is, 28- and 35-dai samples were pooled, and 42- and 49-dai samples were similarly pooled. After pearling, the bran and nonbran fractions were separately analyzed for DON concentration, using 4-g samples. DON percentage in the nonbran fraction was calculated on the basis of bran constituting $14.5 \%$ of total kernel weight, with the nonbran fraction accounting for the remaining $85.5 \%$ (National Festival of Breads 2020).

Assays of fungal DNA. Using quantitative polymerase chain reaction (qPCR), three sets of samples were assayed for levels of $F$. $g r a-$ minearum DNA associated with different infection timings and at different stages of spike maturity. Fungal DNA was presumed to correlate with fungal biomass. Two of the sample sets were chosen to measure the effects of early versus late infection on the quantity of fungal DNA at intervals during grain-fill: (i) the 0- and 7-daa timings from 2010, for which the 14-, 21-, 28-, 35-, and 42-dai samples were assayed; and (ii) the 3- and 13-daa samples from 2012, for which the 21-, 28-, 35-, 42-, and 49-dai samples were assayed. Three (2010) or four (2012) replicate samples per daa $\times$ dai combination were 
analyzed. In the third set of samples, the 42- and 49-dai samples for all infection timings in 2012 were assayed in order to compare fungal biomass at a similar timepoint (physiological maturity).

The qPCR was conducted as described in Beccari et al. (2019). To set up the standard curve, DNA extraction from pure fungal culture and from healthy kernels of winter wheat was conducted preliminarily. F. graminearum strain $\mathrm{J}-2$ was grown on potato dextrose agar (BD, Franklin Lakes, NJ) for 1 week prior to DNA extraction. Mycelium was scraped using a spatula, placed in a 2-ml plastic tube (Eppendorf North America, Hauppauge, NY) and freeze dried. After the addition of a steel bead for each tube, fungal tissues were finely ground (1 min) by Vortex Genie 2 (Daigger, Vernon Hills, IL). DNA was extracted following the protocols described by Covarelli et al. (2015).

Uncontaminated kernels of winter wheat cultivar NC-Neuse were finely ground in a laboratory blender, and DNA was extracted by the method described by Parry and Nicholson (1996) with some modifications described in Beccari et al. (2019). The quality of extracted DNA from the $F$. graminearum strain J-2 and from healthy winter wheat kernels was estimated by DS-11 spectrophotometer (Denovix, Wilmington, DE), and the concentration of isolated DNA was measured by Qubit 2.0 fluorimeter (Invitrogen, Life Technologies, Thermo Fisher Scientific, Waltham, MA) using a Qubit dsDNA BR assay kit (molecular probes, Life Technologies, Thermo Fisher Scientific) following the manufacturer's instructions.

A dilution series from $0.05 \mathrm{pg}$ to $50 \mathrm{ng}$ of $F$. graminearum strain $\mathrm{J}$ 2 DNA and from $5 \mathrm{pg}$ to $50 \mathrm{ng}$ of winter wheat DNA, with a serial dilution factor of 10 , was produced to set up standard curves that were processed in each qPCR assay. Two replicates of each standard were used in each assay. Standard curves were generated by plotting the logarithmic values of known DNA quantities versus the corresponding cycle threshold $(\mathrm{Ct})$ values. For each standard curve, from the average $\mathrm{Ct}$ of each dilution, the equation $y=m x+q$ was calculated as well as the $R^{2}$ value and reaction efficiency $\left(10^{(-1 / m)}\right)$.

$R^{2}$ values calculated from the linear equations of the two standard curves were 0.988 for soft wheat and 0.991 for $F$. graminearum. Reaction efficiencies obtained from the linear equations of the two standard curves were $98 \%$ for soft wheat and $97 \%$ for $F$. graminearum. The limit of detection of $F$. graminearum biomass was $0.05 \mathrm{pg}$.

Total DNA from the samples, including winter wheat DNA and possibly infected $F$. graminearum DNA, was isolated using the method described above for the uncontaminated winter wheat kernels. Quality and concentration of extracted DNA were estimated as previously described, and the concentration of each DNA sample was adjusted to $20 \mathrm{ng} / \mu \mathrm{l}$ for $\mathrm{qPCR}$ analyses. The specific primers were as follows: for the detection and quantification of $F$. graminearum, Fg16N F (5'ACAGATGACAAGATTCAGGCACA-3') and Fg16N R (5'-TTCT TTGACATCTGTTCAACCCA-3') (Brandfass and Karlovsky 2008); for the quantification of winter wheat DNA, translation elongation factor $1 \alpha(T E F 1 \alpha)$ primers Horl $\mathrm{F}\left(5^{\prime}\right.$-TCTCTGGGTTTGAGGGT GAC-3') and Hor1 R (5'-GGCCCTTGTACCAGTCAAGGT-3') (Nicolaisen et al. 2009).

To optimize qPCR reaction conditions, annealing temperatures (from 55 to $65^{\circ} \mathrm{C}$ ) were adjusted experimentally. The qPCR assays were carried out in a CFX96 Real-Time System (Bio-Rad, Hercules,

Table 1. Temperature and precipitation during the anthesis and grain-fill periods of four years when the window of susceptibility to Fusarium head blight was monitored in winter wheat cultivars in North Carolina ${ }^{\mathrm{a}}$

\begin{tabular}{|c|c|c|c|c|c|c|}
\hline \multirow[b]{3}{*}{ Year } & \multicolumn{6}{|c|}{ Weather variables during periods after 0 daa } \\
\hline & \multicolumn{3}{|c|}{$\begin{array}{l}\text { Temperature (mean of daily } \\
\left.\text { mean }{ }^{\circ} \mathrm{C}\right)\end{array}$} & \multicolumn{3}{|c|}{ Total precipitation $(\mathrm{cm})$} \\
\hline & 14 days & 21 days & 42 days & 14 days & 21 days & 42 days \\
\hline 2010 & 20.7 & 20.5 & 21.2 & 1.0 & 1.0 & 20.0 \\
\hline 2011 & 19.0 & 18.8 & 21.1 & 2.6 & 3.5 & 9.2 \\
\hline 2012 & 15.5 & 15.7 & 18.1 & 2.1 & 2.4 & 10.7 \\
\hline 2013 & 15.4 & 16.6 & 19.3 & 7.0 & 7.1 & 13.0 \\
\hline
\end{tabular}

a daa $=$ days after anthesis.
CA). The qPCR mixture was composed of a total reaction volume of $12 \mu \mathrm{l}$, containing $2.5 \mu \mathrm{l}$ of total DNA, $6 \mu \mathrm{l}$ of $2 \times$ SsoFast EvaGreen Supermix (Bio-Rad), $1.5 \mu \mathrm{l}$ of $2 \mu \mathrm{M}$ each primer, and $0.5 \mu \mathrm{l}$ of sterile DNase-free water (Thermo Fisher Scientific). The program consisted of $50^{\circ} \mathrm{C}$ for $2 \mathrm{~min}, 95^{\circ} \mathrm{C}$ for $10 \mathrm{~min}, 45$ cycles at $95^{\circ} \mathrm{C}$ for $15 \mathrm{~s}$ and specific annealing temperature of each primer $\left(60^{\circ} \mathrm{C}\right.$ for $\mathrm{Fg} 16 \mathrm{~N}$ and $58^{\circ} \mathrm{C}$ for Hor 1) for $1 \mathrm{~min}$, heating at $95^{\circ} \mathrm{C}$ for $10 \mathrm{~s}$, cooling at $60^{\circ} \mathrm{C}$, and finally an increase to $95^{\circ} \mathrm{C}$ at $0.5^{\circ} \mathrm{C}$ every $5 \mathrm{~s}$ with the measurement of fluorescence. A dissociation curve was included at the end of the qPCR program to monitor the presence of potential primer-dimers and nonspecific amplification products. Two replicates of each sample were used in each assay. The $F$. graminearum biomass in the winter wheat grains was expressed as the ratio of the fungal DNA (pg) to the plant DNA (ng).

Statistical analyses. In general, mean values of DON, FDK, and PIK were compared using the standard errors of the means; where noted, $t$ tests were performed. To evaluate the ratios of DON to FDK, PROC MIXED of SAS version 9.4 (SAS Institute, Cary, NC) was used to model the ratios as a function of daa and cultivar, and least-square means were compared.

\section{Results}

Environmental conditions. During the period from 0 to 42 daa, important weather variables differed among the four experimental years (Table 1, Fig. 1). Most notably, in 2010 and 2011, the mean temperature in the 14- and 21-day periods after 0 daa was 3 to $5^{\circ} \mathrm{C}$ higher than in 2012 or 2013 (Table 1). In 2012, there were cold snaps from 1 to 3 daa and from 12 to 15 daa when the mean daily temperature dropped to 8 to $13^{\circ} \mathrm{C}$ for 3 and 4 days, respectively (Fig. 1). Total precipitation in the 14- and 21-day periods after 0 daa was also lowest in 2010, intermediate in 2011 and 2012, and substantially higher in 2013. Thus, the 2010 and 2011 trials took place under hotter, drier conditions than the 2012 and 2013 trials, and 2013 was the highest-rainfall year.

Unsurprisingly, the dry conditions in 2010 accelerated wheat development by about 2 days over 2012 (Table 2). For example, the cultivars reached kernel watery ripe stage by 7 daa in 2010 but not until 9 daa in 2012.

2010 (dry year). Index (disease symptoms). Mean levels of visually rated disease symptoms were considerably higher after 0 -daa inoculations than after any later inoculation for both NC-Neuse and P26R12 (Fig. 2A). As expected, the mean index for the susceptible P26R12 was significantly higher than for the moderately resistant NC-Neuse for 0 -daa infections ( $t$ test, $P=0.003$ ). The index levels from the 13-, 15-, 17-, 19-, and 21-daa inoculations were all zero and are not shown.

$D O N, P I K$, and FDK. The highest mean DON levels were attained in the 0 -daa treatments for both cultivars during anthesis and grainfill (Fig. 2B and C; note different vertical scales). The highest DON levels attained during grain-fill were $\sim 3.5$ to $5 \mu \mathrm{g} / \mathrm{g}$ from 28 to 35 dai for NC-Neuse (Fig. 2B) and $\sim 11 \mu \mathrm{g} / \mathrm{g}$ at 28 dai for P26R12 (Fig. 2C). Mean DON levels in samples from the 7-daa inoculated plots were higher than those from the 9- or 11-daa inoculated plots for both cultivars at most sampling points after 14 dai. For those 9- and 11-daa inoculated treatments, mean DON stayed at or below $2 \mu \mathrm{g} / \mathrm{g}$ throughout grain-fill. No mean DON level was higher than $0.8 \mu \mathrm{g} / \mathrm{g}$ for NC-Neuse and $1.3 \mu \mathrm{g} / \mathrm{g}$ for P26R12 for any later-inoculated treatment (data not shown).

Fusarium-infected kernels developed substantially only from the 0 - and 7-daa infection timings for both NC-Neuse (Fig. 2D) and P26R12 (Fig. 2E), and then only late in grain-fill, about a month after infection. Considering the 0-daa infection timing, P26R12 developed a little less than twice the PIK as NC-Neuse at the last sampling point (41 versus 24\%). The 7-daa PIK level was intermediate for both cultivars. From the later infection timings, lower levels of PIK developed for both cultivars, fluctuating as high as approximately $5 \%$ for NC-Neuse and $10 \%$ for P26R12.

At physiological maturity in 2010, DON levels were higher for samples from the 0-daa inoculated treatments of both cultivars than for all later inoculation timings (Fig. 2F). Final DON levels were 5.7 and $13.5 \mu \mathrm{g} / \mathrm{g}$ for NC-Neuse and P26R12, respectively, for 
inoculations at 0 daa. Inoculations at 7 daa resulted in a harvest-time DON level of $0.3 \mu \mathrm{g} / \mathrm{g}$ for NC-Neuse, and all subsequent infection timings led to harvest-time DON levels below that. However, in the susceptible cultivar P26R12, inoculations at 7 and 9 daa led to harvest-time DON concentrations of 3.7 and $1.9 \mu \mathrm{g} / \mathrm{g}$, respectively (values not significantly different, $P>0.05$ ). Thus, the window for infections leading to DON levels above the commercial ceiling of $2 \mu \mathrm{g} / \mathrm{g}$ at harvest was less than 7 days for NC-Neuse but at least 7 days for P26R12.

Kernel damage at physiological maturity in 2010 followed a pattern similar to DON, with FDK highest from 0-daa infections (Fig. $2 \mathrm{G})$. Weights of 30-spike samples generally reflected the FDK data, except the differences for NC-Neuse were less (Fig. 2G). Mean FDK levels in both cultivars were 13 to $19 \%$ from 0-daa infections, whereas all subsequent infection timings resulted in lower FDK levels. The two cultivars did not differ significantly in FDK from the 0-daa infections $(P=0.16)$, despite the fact that P26R12 had nearly twice the PIK of NC-Neuse, suggesting that P26R12 had a higher level of plump, asymptomatic kernels containing Fusarium. Although 7-daa and later infections led to very little FDK in NC-Neuse, that was only true for 13-daa and later infections in P26R12, indicating that P26R12 was susceptible to FDK longer than NC-Neuse. Mean FDK levels in P26R12 were 2.7 to $4.0 \%$ from 7- and 9-daa infections and $0.5 \%$ in 11-daa infections.

Fungal DNA. In 2010, the dry year, $F$. graminearum DNA concentrations in both cultivars were generally higher in the treatment inoculated at 0 daa than in the treatment inoculated at 7 daa, indicating greater fungal biomass in the early-infected samples than in the later-infected samples (Fig. 2H). The early-infected concentrations were higher at nearly all timepoints during grain-fill, including at physiological maturity in early June. Particularly in the earlyinfected samples of P26R12, the susceptible cultivar, the amount

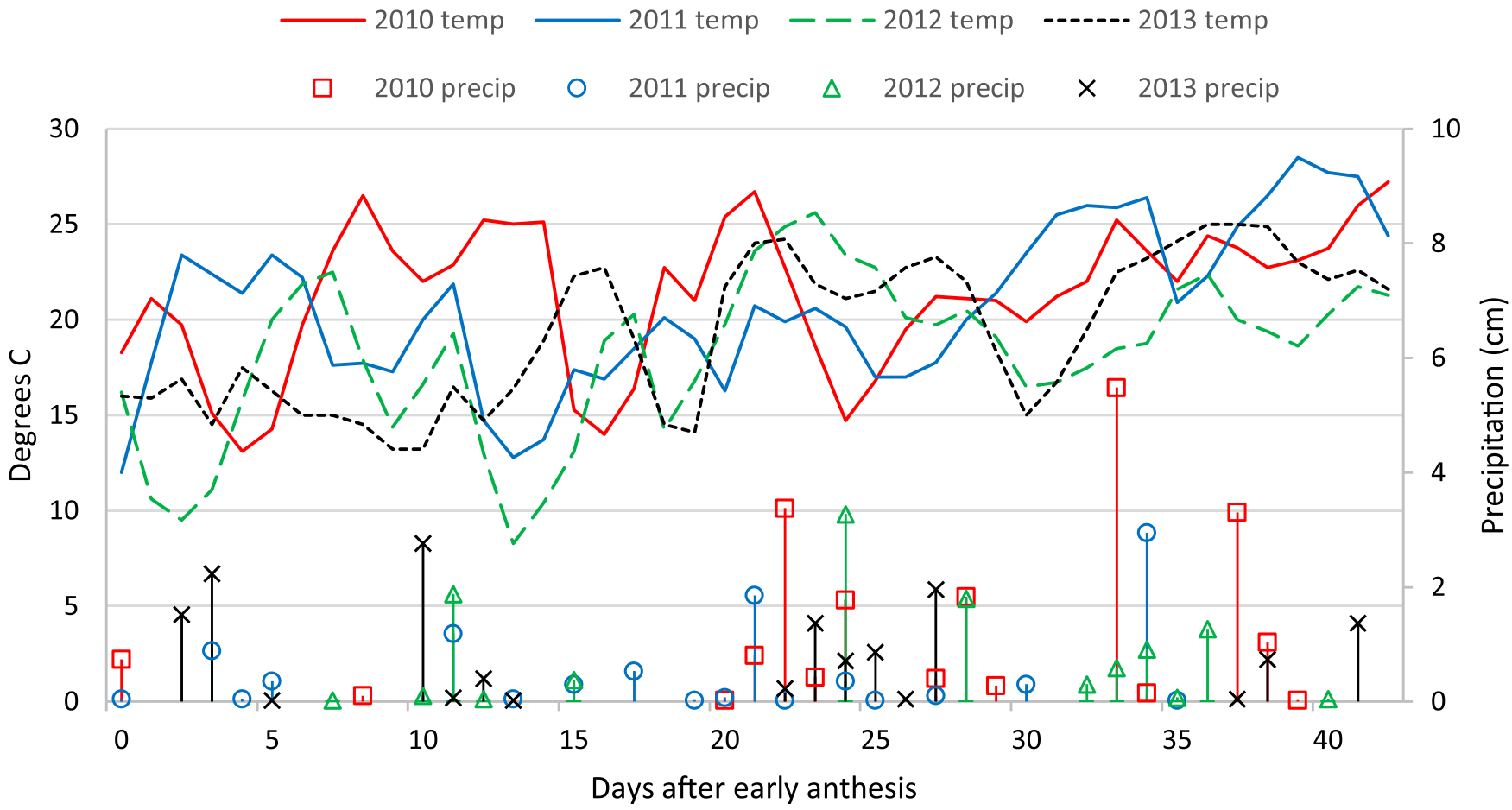

Fig. 1. Daily average 2-m air temperature and daily precipitation during the 42 days after the start of anthesis in the 4-year experiment on the duration of susceptibility to Fusarium head blight in winter wheat at Raleigh, North Carolina.

Table 2. Wheat growth stages as determined by spike dissections at various inoculation timings in an experiment on the duration of susceptibility to Fusarium head blight using two winter wheat cultivars in North Carolina ${ }^{\mathrm{a}}$

\begin{tabular}{|c|c|c|c|c|c|c|c|c|c|}
\hline \multirow[b]{3}{*}{ Infection timing } & \multicolumn{3}{|c|}{2010} & \multicolumn{3}{|c|}{2011} & \multicolumn{3}{|c|}{2012} \\
\hline & \multicolumn{2}{|c|}{ Cultivar } & \multirow[b]{2}{*}{ Growth stage } & \multicolumn{2}{|c|}{ Cultivar } & \multirow[b]{2}{*}{ Growth stage } & \multicolumn{2}{|c|}{ Cultivar } & \multirow[b]{2}{*}{ Growth stage } \\
\hline & NC-Neuse & P26R12 & & NC-Neuse & P26R12 & & NC-Neuse & P26R12 & \\
\hline 0 daa & 63.3 & 61.9 & Early anthesis & 66.2 & & Midanthesis & 65.3 & 65.2 & Midanthesis \\
\hline 3 daa & & & & 68.5 & 68.8 & Anthesis complete & 68.7 & 67.0 & Late anthesis \\
\hline 5 daa & & & & 70.2 & 68.8 & Late anthesis & 68.3 & 67.5 & Late anthesis \\
\hline 6 daa & & & & 72.3 & 70.7 & Kernel watery ripe & 69.0 & 68.5 & Anthesis complete \\
\hline 7 daa & 70.6 & 70.7 & Kernel watery ripe & 72.7 & 72.8 & Early milk & 69.8 & 70.0 & \\
\hline 8 daa & & & & 73.0 & 72.7 & Early milk & 70.3 & 70.3 & \\
\hline 9 daa & 71.0 & 71.2 & Kernel watery ripe & 73.0 & 73.0 & Early milk & 70.8 & 71.5 & Kernel watery ripe \\
\hline 11 daa & 73.0 & 72.8 & Early milk & 74.9 & 74.7 & Medium milk & 71.5 & 72.1 & Kernel watery ripe \\
\hline 13 daa & 76.7 & 75.0 & Medium to late milk & & 77.3 & Late milk & 72.5 & 73.5 & Early milk \\
\hline 15 daa & 77.3 & 76.1 & Medium to late milk & & & & & & \\
\hline 17 daa & 83.0 & 78.3 & Late milk to early dough & & & & & & \\
\hline 19 daa & 85.0 & 84.0 & Soft dough & & & & & & \\
\hline 21 daa & 87.0 & 87.0 & Hard dough & & & & & & \\
\hline
\end{tabular}

a Numerical values $=$ Zadoks scale for growth and development of cereals. NC-Neuse was moderately resistant to Fusarium head blight; P26R12 was susceptible. daa $=$ days after anthesis. 
of $F$. graminearum DNA increased sharply at the final sampling point, on 4 June, indicating that the fungus was still spreading in the spikes. Overall, early-infected P26R12 had much higher fungal DNA concentrations than early-infected NC-Neuse, with the sole exception of the 28 May P26R12 sample. Late-infected P26R12 had similar levels of fungal DNA to early-infected NC-Neuse.
2011. Index. As in 2010, mean levels of visually rated disease symptoms were substantially higher after 0-daa inoculations than after any later inoculation for both NC-Neuse and P26R12 (Fig. 3A) Only inoculations at 0 daa (NC-Neuse) or 0 and 3 daa (P26R12) produced index levels significantly higher than the control for either cultivar. Again, the mean index for the susceptible P26R12 was
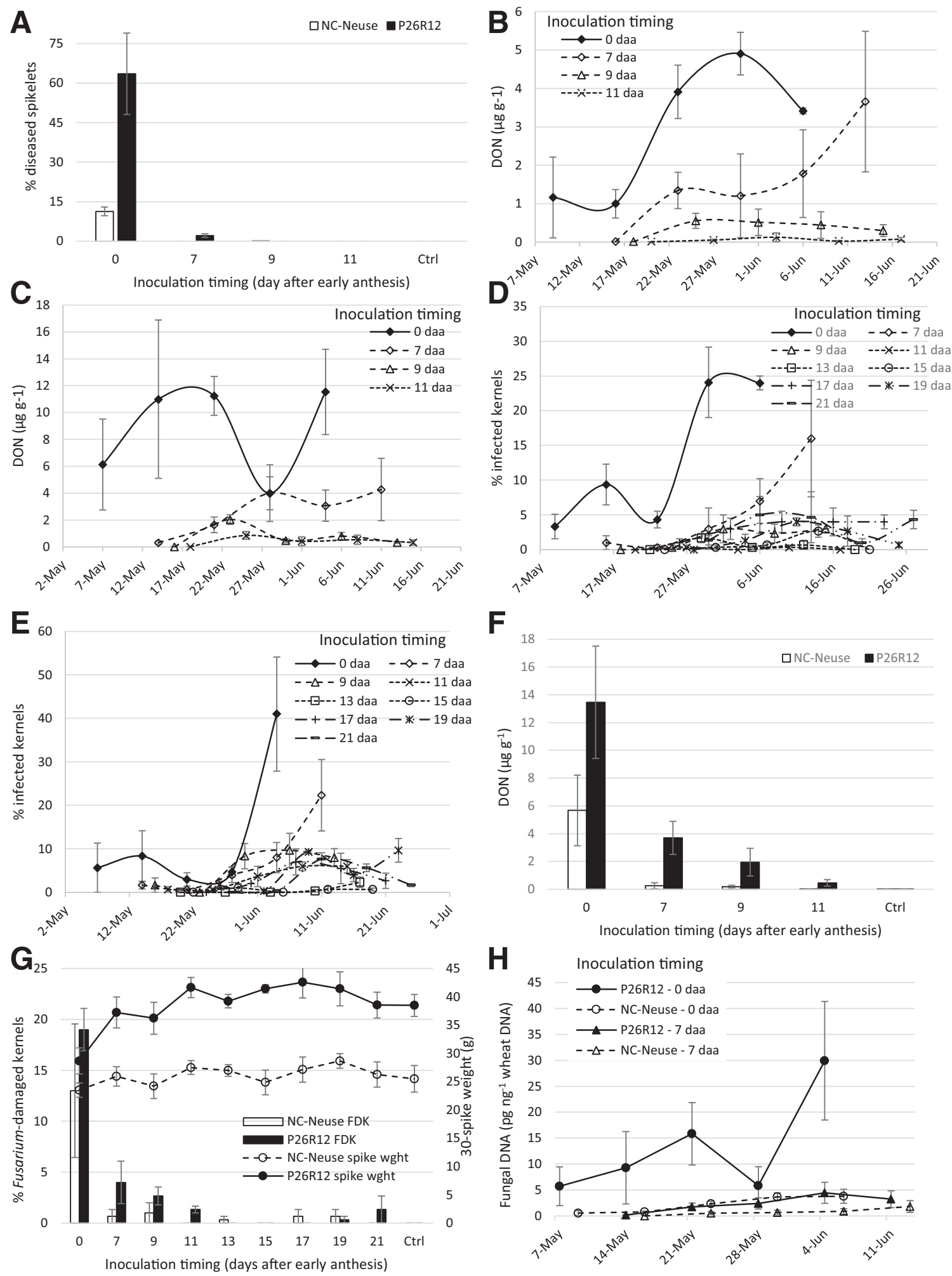

Fig. 2. Susceptibility of winter wheat to Fusarium graminearum infection at varying numbers of days after early anthesis (daa) in 2010 . A, Percent diseased spikelets (incidence $\times$ severity/100) in moderately resistant cultivar NC-Neuse and susceptible cultivar P26R12. At five time points during grain-fill, deoxynivalenol (DON) in B, NC-Neuse, and C, P26R12, and percent infected kernels in D, NC-Neuse, and E, P26R12. In both cultivars at physiological maturity on 10 June, F, DON, G, Fusarium-damaged kernels and 30-spike weights, and $\mathbf{H}$, fungal DNA concentrations in 0 - versus 7-daa inoculated spikes. Values are means of three replicates; error bars indicate the standard error of the mean; Ctrl = noninoculated control; and 0 daa $=23$ April (P26R12) or 25 April (NC-Neuse). 
significantly higher than for the moderately resistant NC-Neuse for 0 -daa infections ( $t$ test, $P<0.0001$ ).

$D O N$ and FDK. During grain-fill, the maximum concentration of DON was similar in NC-Neuse to what it had been in 2010 (Fig. 3B). The 0-, 3-, and 11-daa treatments all spiked briefly to 3 to $7 \mu \mathrm{g} / \mathrm{g}$, but otherwise levels remained under $3 \mu \mathrm{g} / \mathrm{g}$ in NC-Neuse throughout the sampling period, and standard errors were high. In the susceptible cultivar P26R12, by contrast, the 0 -daa treatment had $35 \mu \mathrm{g} / \mathrm{g}$ DON at 21 dai, and it had higher DON than all other treatments from 21 dai until harvest (Fig. 3C). All other treatments remained below $10 \mu \mathrm{g} / \mathrm{g}$ throughout the trial.

DON at harvest ripeness reflected the dynamics of the index, in that it was low $(\leq 2 \mu \mathrm{g} / \mathrm{g})$ in NC-Neuse for all inoculation timings,
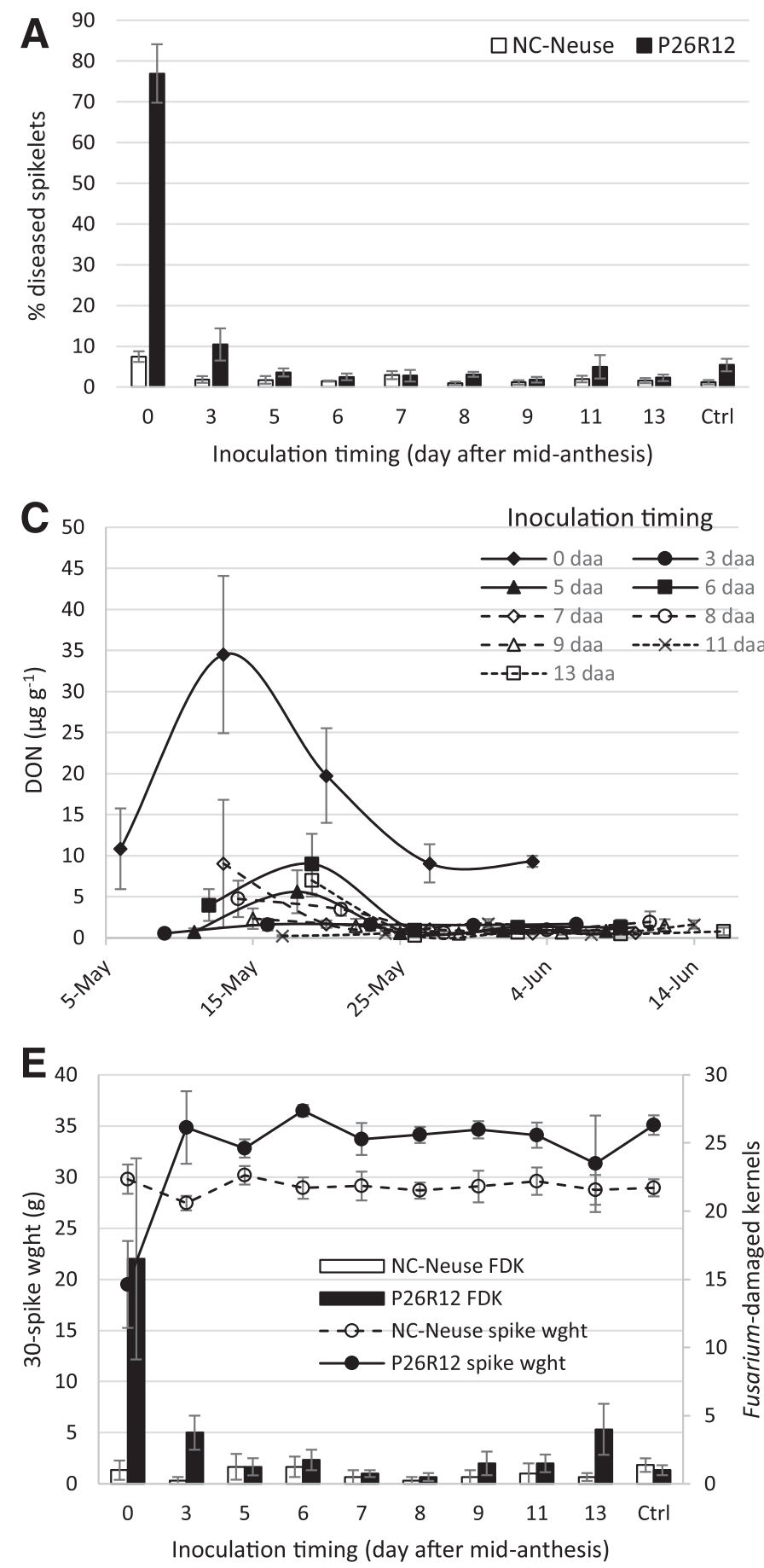

and significantly different from the noninoculated control for the 0 -daa timing $(P=0.03)$ but not from other timings $(P \geq 0.47)$ (Fig. 3D). DON at harvest was higher in P26R12 from the 0-daa inoculation timing than for any other timing. In P26R12, unlike in NCNeuse, the 13-daa inoculation produced DON that was at the $\sim 2 \mu \mathrm{g} / \mathrm{g}$ threshold despite the late timing of infection.

For P26R12, kernel damage at harvest reflected the DON pattern, with FDK highest and 30-spike weights lowest from 0-daa infections (Fig. 3E). NC-Neuse exhibited very low levels of FDK, and 30-spike weights were little affected, although 3-daa weights were slightly lower than those from 0-daa infections.

2012. As mentioned in the Materials and Methods, irrigation was erroneously not applied immediately after the 0-daa inoculation; it
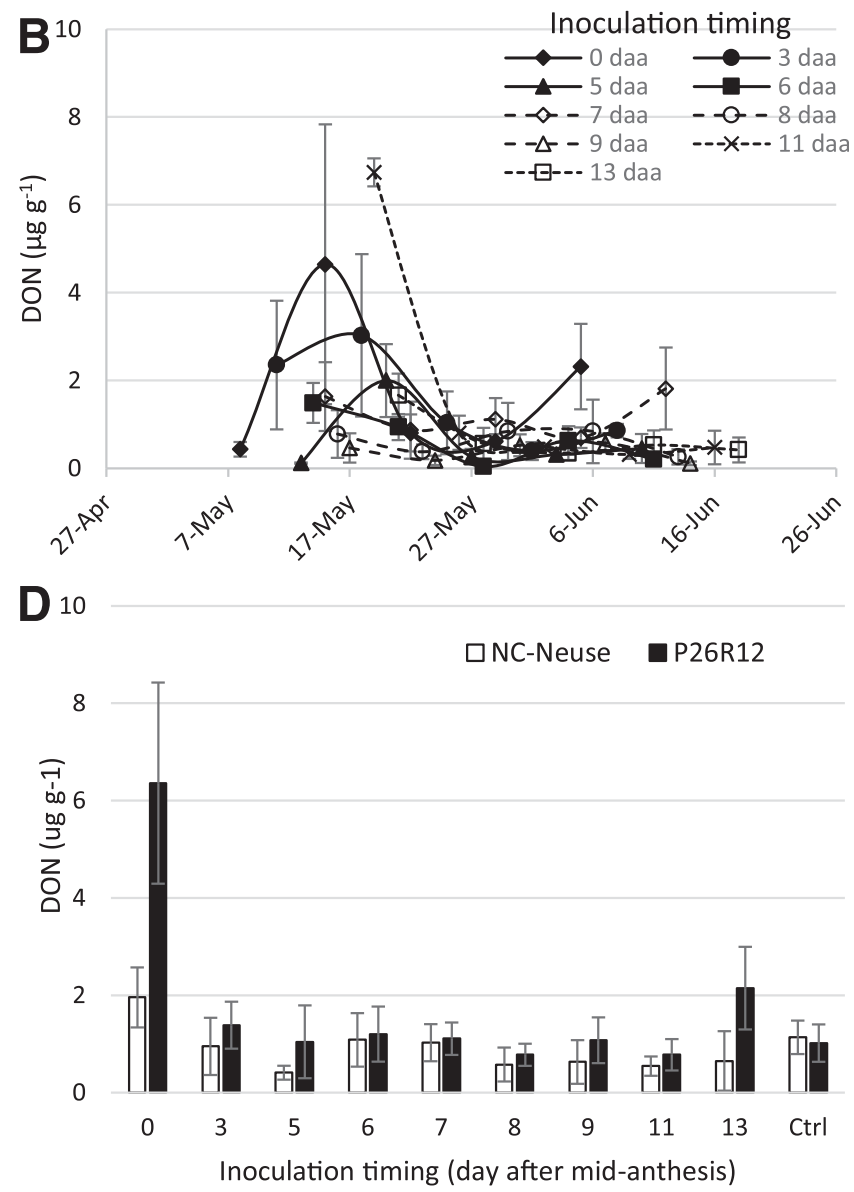

Fig. 3. Susceptibility of winter wheat to Fusarium graminearum infection at varying numbers of days after early anthesis (daa) in 2011 . A, Percent diseased spikelets (incidence $\times$ severity/100) in moderately resistant cultivar NC-Neuse and susceptible cultivar P26R12. At five time points during grain-fill, deoxynivalenol (DON) in B, NC-Neuse, and C, P26R12. In both cultivars at physiological maturity on 20 June, D, DON, and E, 30-spike weight and Fusarium-damaged kernels. Values are means of four replicates; error bars indicate the standard error of the mean; Ctrl = noninoculated control; and 0 daa = 22 April (P26R12) or 24 April (NC-Neuse). 
started at the 3-daa inoculation. The 0-daa results are presented nevertheless for completeness. Brief but severe cold temperature snaps occurred during the inoculation period (Fig. 1) and are considered in detail in the Discussion.

Index. The window of infection timings producing visual symptoms was wider in 2012 (Fig. 4A) than in 2010, although the maximum index achieved was lower for P26R12 and higher for NC-Neuse than in 2010. For P26R12, the highest levels of visual symptoms were from 3- and 9-daa inoculations, whereas for NC-Neuse, the highest levels of visual symptoms were from 3-, 5-, 6-, and 9-daa inoculations. Although the two cultivars had similar levels of index from the 0-, 5-, and 6-daa infections, P26R12 had more symptoms than NC-Neuse from later inoculations $(3,7,9$, and $11 \mathrm{daa})$. Thus, the greater susceptibility of P26R12 was more evident in the later part of anthesis than in the earlier part.

$D O N, P I K$, and FDK. DON levels were much higher during grainfill in 2012 than in 2010 (Fig. 4B and C). Starting early in grain-fill, the 7-daa inoculation produced the highest level of DON in NCNeuse (Fig. 4B), despite the relatively minor symptoms in NCNeuse from that infection timing (Fig. 4A). The 9-daa inoculation resulted in relatively high DON levels during grain-fill in P26R12 (Fig. 4C), which was reflective of the high disease severity from that infection timing. The highest DON level of $64 \mu \mathrm{g} / \mathrm{g}$ in the NC-Neuse 7-daa treatment was from 14 dai (Fig. 4B), but the comparable 14-dai sample for the 9-daa treatment in P26R12 was unavailable; data are presented for that treatment starting at 21 dai (Fig. 4C). For NCNeuse, the 0-, 11-, and 13-daa inoculations were all relatively low DON throughout grain-fill. For P26R12, the 0-daa inoculation was relatively low DON throughout, possibly in part because of the lack of early irrigation, whereas other inoculation timings fluctuated during grain-fill; even the latest infection timings of 11 and 13 daa produced as much as 10 to $12 \mu \mathrm{g} / \mathrm{g}$ at points during grain-fill.

PIK rose steadily during grain-fill until it plateaued at the end of May (Fig. 4D and E). This reflects the relatively constant spread of the pathogen in spikes, which was much more extensive in 2012 than in the drier year of 2010. Maximum levels of PIK in 2012 were 70 to $80 \%$ for NC-Neuse (Fig. 4D) and 75 to 85\% for P26R12 (Fig. 4E). For NCNeuse, most inoculation timings produced similar PIK throughout grainfill, but 3- and 9-daa inoculations resulted in higher levels at the end of the sampling period, whereas 0- and 13-daa inoculations resulted in lower levels starting at around 10 May (Fig. 4D). For P26R12, the 9-daa inoculation produced generally high levels of PIK (Fig. 4E), in keeping with the DON levels from that inoculation (Fig. 4C). In the case of P26R12, the susceptible cultivar, unlike NC-Neuse, even the 13-daa inoculation led to PIK levels similar to those from the remaining inoculation timing.

At harvest, NC-Neuse and P26R12 had similar DON levels for all infection timings through 7-daa; however, for 9-, 11-, and 13-daa inoculations, P26R12 had higher final DON concentrations than NCNeuse (Fig. 4F). For FDK, the highest percentages in NC-Neuse were 3- to 6- and 9-daa inoculations, whereas from P26R12 they were from 3- and 9-daa inoculations (Fig. 4G). Spike weights closely reflected the FDK pattern (Fig. 4G).

Fungal DNA. F. graminearum DNA concentrations near physiological maturity were compared by assaying samples of all infection timings collected between 29 May and 4 June (Fig. 4H). DNA concentrations in the two cultivars were not significantly different from inoculations from 0 to 5 daa $(P \geq 0.06)$. However, considering progressively later infection timings, the higher amount of fungal DNA was more frequently observed in the susceptible cultivar P26R12, which had significantly more fungal DNA in the 6-, 8-, 9-, 11-,
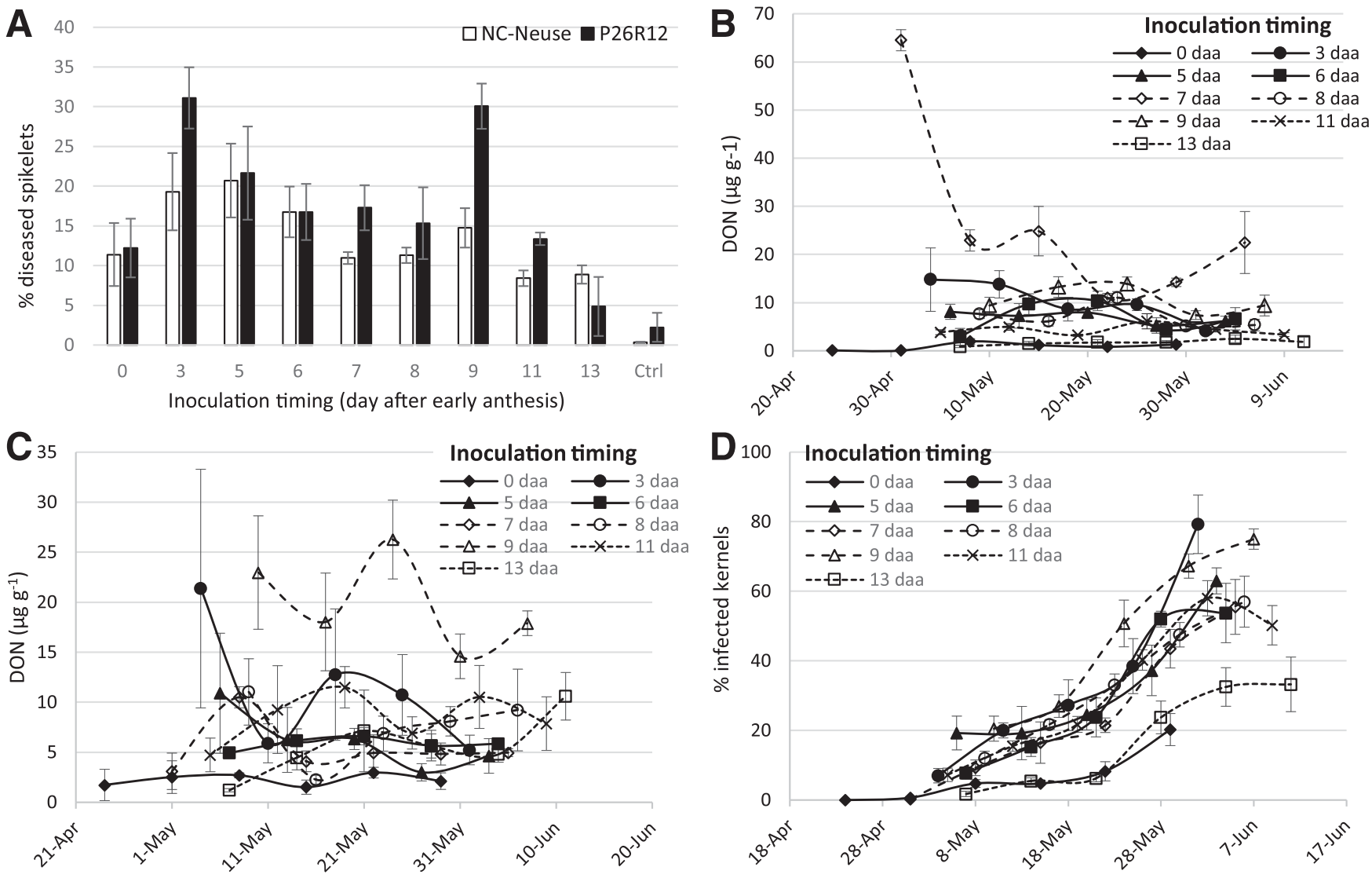

(Continued)

Fig. 4. Susceptibility of winter wheat to Fusarium graminearum infection at varying numbers of days after midanthesis (daa) in 2012. A, Percent diseased spikelets (incidence $\times$ severity/100) in moderately resistant cultivar NC-Neuse and susceptible cultivar P26R12. At six points during grain-fill, deoxynivalenol (DON) in B, NC-Neuse, and C, P26R12, and percent infected kernels in D, NC-Neuse, and E, P26R12. At physiological maturity, 29 May to 4 June, F, DON, G, Fusarium-damaged kernels (FDK) and 15-spike weight, H, fungal DNA, and I, progression of fungal DNA from early and late infections. Values = means of four replicates for NC-Neuse, three for P26R12; error bars = standard error of the mean; $\mathrm{Ctrl}=$ noninoculated control; and 0 daa $=10$ April, both cultivars. 
and 13-daa infected plots $(P \leq 0.049)$. This was consistent with the pattern of P26R12 having a wider window of susceptibility to $F$. graminearum infection than NC-Neuse.

Although $F$. graminearum spread in spikes as evidenced by DNA concentrations had similar dynamics to those in 2010, fungal DNA concentrations were overall substantially higher in the cooler and somewhat wetter year of 2012 (Fig. 4I). When infected at 3 daa, both cultivars attained higher maximum levels of fungal DNA than when infected at 13 daa. In 2012, P26R12 samples that were infected early (3 daa) had fungal DNA concentrations in late May over twice as high as the highest concentration in 2010, but the level did not increase after 18 May, suggesting that $F$. graminearum had reached the limit of its ability to spread. By contrast, the early-infected
NC-Neuse and even the late-infected P26R12 had increasing fungal DNA concentrations in late May and early June, indicating that the fungus was still spreading in those spikes during the last part of grain-fill. Although they increased slightly, DNA concentrations in late-infected NC-Neuse remained relatively low until harvest.

2013 (wet year). Index. As in 2010 and 2011, and unlike 2012, blight symptoms were confined to infections from the relatively early part of anthesis (Fig. 5A). Symptoms were caused by the 0- and 3-daa inoculations in both cultivars but by the 5- and 6-daa inoculations only in the susceptible P26R12. In P26R12, symptoms were significantly more severe from the 3-daa inoculations $(51 \%)$ than from any other infection timing $(P \leq 0.008)$ and were comparable to the $64 \%$ from the 0-daa inoculation in 2010.

Fig. 4. (Continued from previous page)

E 100 Inoculation timing
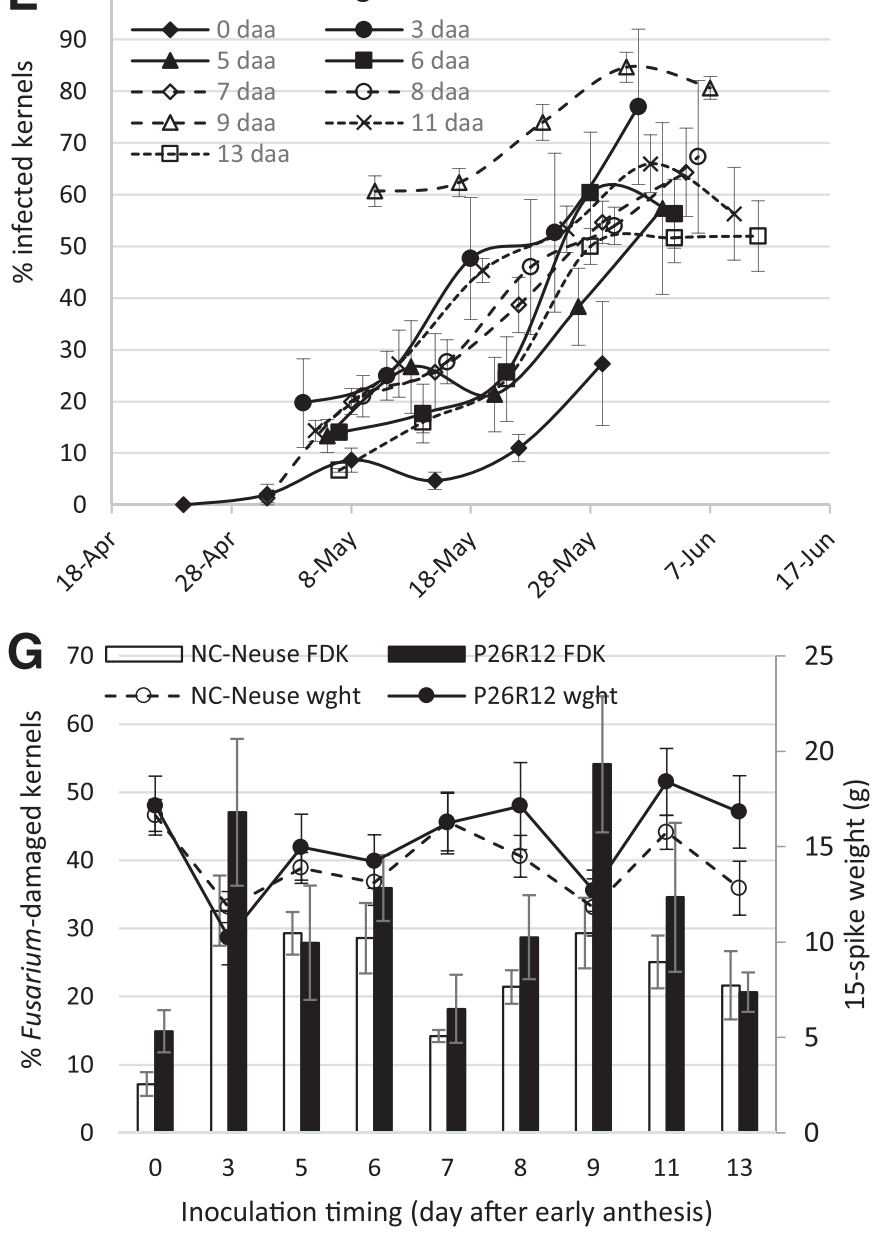

I Inoculation timing

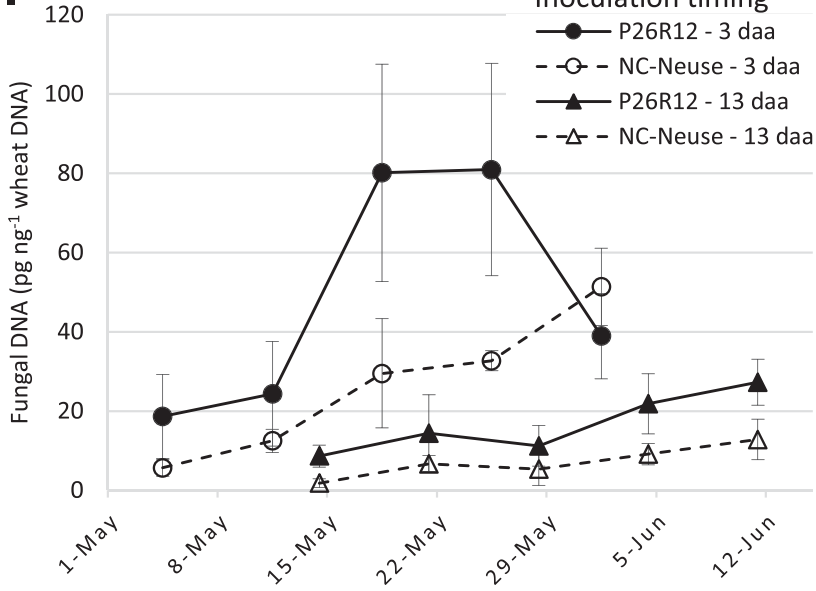

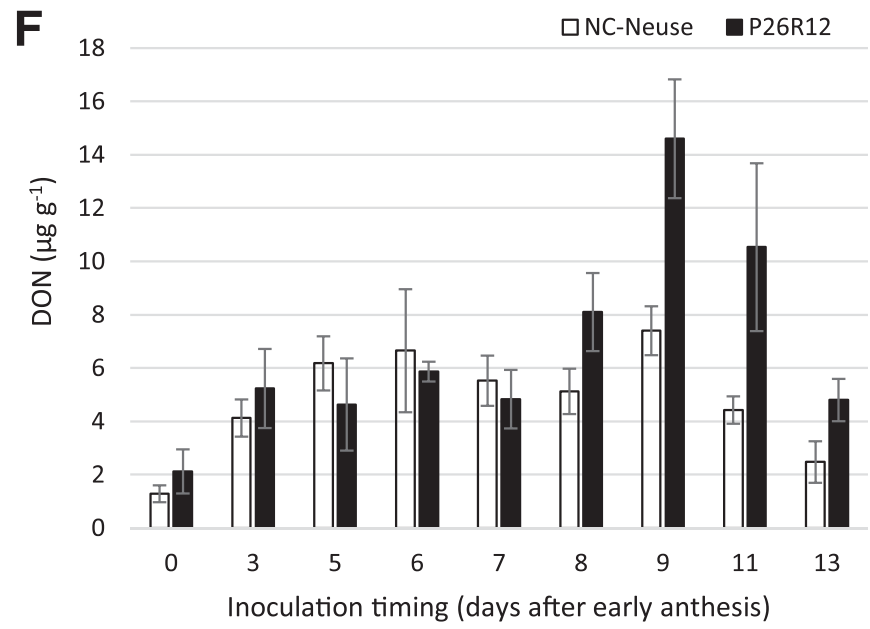

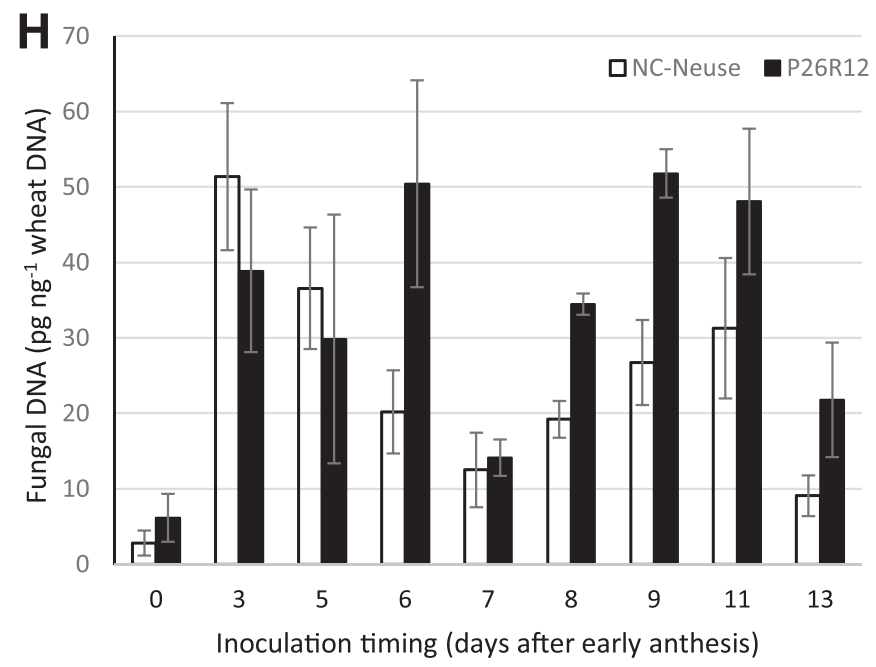


DON and FDK. In 2013, the maximum DON levels observed during grain-fill were much higher in both cultivars than in 2010, and in P26R12 the maximum level was higher than in 2011 or 2012 (Fig. 5B and $\mathrm{C}$; note different $y$-axis scales). DON concentrations tended to be higher toward the beginning of grain-fill and decline toward the end, although that trend was most pronounced in the earliest-infected treatments.

The 0- and 3-daa inoculations resulted in relatively high DON levels at 14 and 21 dai (Fig. 5B and C). Although 0- and 3-daa infections initially led to similar DON levels in NC-Neuse (Fig. 5B), infection at 3 daa resulted in the highest DON level in P26R12, much higher than 0 daa (Fig. 5C). These maximal, early DON concentrations in
NC-Neuse from the 0 - and 3-daa inoculations were about $25 \mu \mathrm{g} / \mathrm{g}$ in NC-Neuse and were similar in the 0-daa treatment for P26R12. But the DON concentration in the 14-dai sample of the P26R12 3daa treatment was $170 \mu \mathrm{g} / \mathrm{g}$ (off the chart in Figure 5C); it fell then by 21 dai to $65 \mu \mathrm{g} / \mathrm{g}$. In P26R12, the DON concentration from the 3 daa treatment remained significantly higher than the DON levels of all other treatments $(P \leq 0.003)$ until the last (42-dai) sample, whereas in NC-Neuse the DON level tended to be higher from the 3-daa inoculation, but differences were not significant $(P>0.05)$.

By contrast, DON levels during grain-fill from all treatments inoculated later than 3 daa fluctuated at or below about $7 \mu \mathrm{g} / \mathrm{g}$ for both cultivars. The 11- and 13-daa treatments produced very little DON
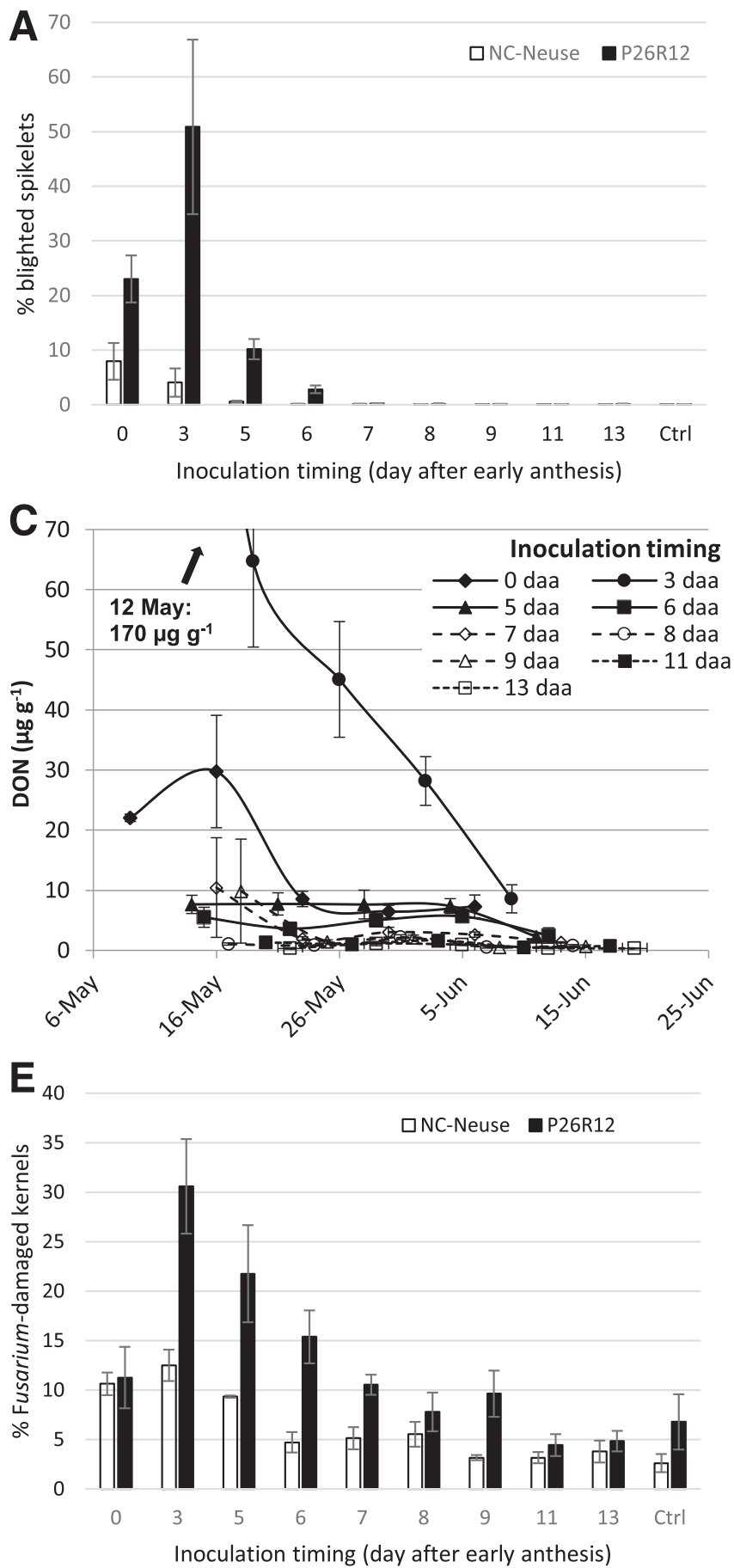
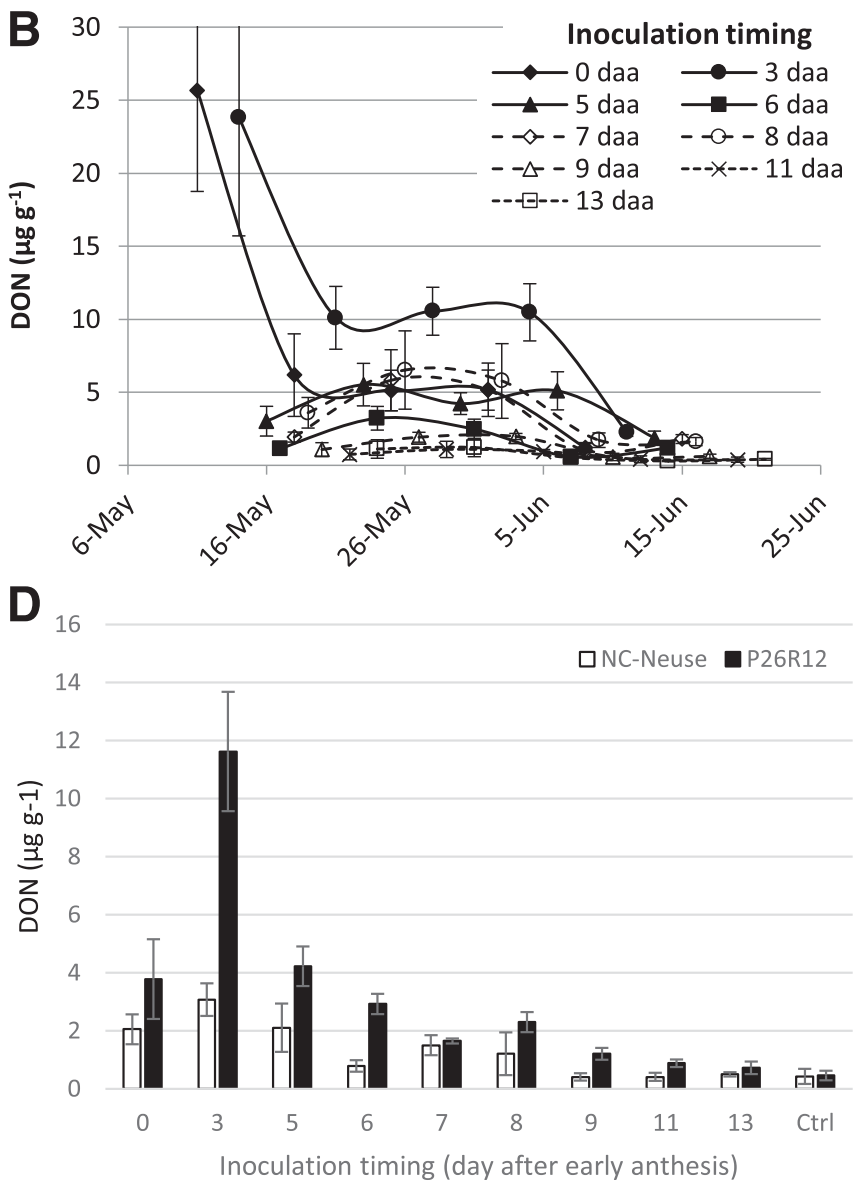

Fig. 5. Susceptibility of winter wheat to Fusarium graminearum infection at varying numbers of days after early anthesis (daa) in 2013. A, Percent blighted spikelets (incidence $\times$ severity/100, or index) in moderately resistant cultivar NC-Neuse and susceptible cultivar P26R12. At five time points during grain-fill, deoxynivalenol (DON) in B, NC-Neuse, and C, P26R12. At physiological maturity on 17 June, D, DON, and E, Fusarium-damaged kernels. Values are means of four replicates; error bars = standard error of the mean; Ctrl = noninoculated control; and 0 daa $=25$ April (P26R12) or 27 April (NC-Neuse). 
in either cultivar at any sampling point (means below $1.3 \mu \mathrm{g} / \mathrm{g}$ throughout grain-fill).

At physiological maturity, mean DON levels were above $2 \mu \mathrm{g} / \mathrm{g}$ for the 0- to 8-daa P26R12 samples (although not for the 7-daa sample), but only for the 0- to 5-daa infected NC-Neuse samples (Fig. 5D). That is, the window of susceptibility to infections resulting in DON levels above $2 \mu \mathrm{g} / \mathrm{g}$ at harvest time was 3 days wider in P26R12 than in NC-Neuse. DON in the 3-daa infected P26R12 was still $>11 \mu \mathrm{g} / \mathrm{g}$ at harvest time.

As in 2010, at physiological maturity FDK levels (Fig. 5E) roughly mirrored DON levels (Fig. 5D) for the various inoculation timings. NC-Neuse had lower levels of FDK than P26R12 for most comparable infection timings. In NC-Neuse, 0 - to 5-daa infections gave rise to the highest levels of FDK, which were above $5 \%$, whereas 0- to 9-daa infections resulted in FDK >5\% in P26R12.
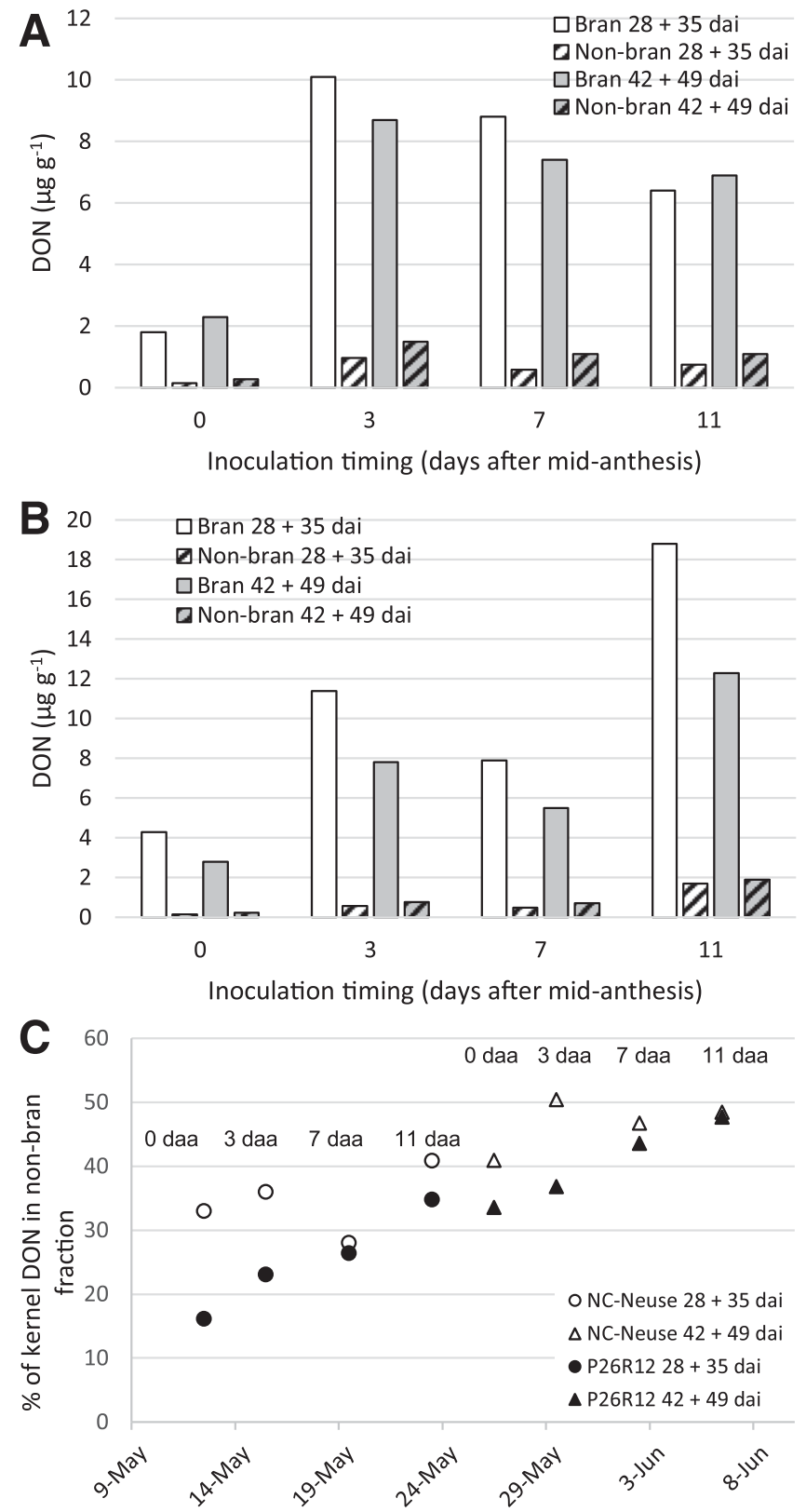

Fig. 6. Deoxynivalenol (DON) concentrations in bran versus nonbran fractions of grain from two winter wheat cultivars, inoculated with Fusarium graminearum at $0,3,7$, or 11 days after early anthesis (daa) and sampled postinoculation (28- and 35-days after inoculation [dai] samples pooled, 42- and 49-dai samples pooled) in 2012. A, Moderately resistant cultivar NC-Neuse, B, susceptible cultivar P26R12, and C, percent of whole-kernel DON in the nonbran fractions, shown by inoculation timing and sampling timing (note the same inoculation timings were sampled successively and are thus represented twice each).
DON in bran versus nonbran. In 2012 samples pearled to separate the bran and nonbran fractions, total kernel DON ranged from 2.0 to $20.5 \mu \mathrm{g} / \mathrm{g}$ (Fig. 6A and B). No confidence intervals could be calculated because treatments were not replicated, due to the need for pooling in order to have sufficient volumes for the pearl mill.

In the pearled samples, the highest DON concentrations were consistently in the bran (Fig. 6A and B). The percentage of DON in the nonbran fraction (i.e., the material from which white flour would be made after bran removal) ranged from 16 to $41 \%$ in the earliercollected samples and 33 to $50 \%$ in the later-collected samples (Fig. 6C). The dates on the $x$ axis in Figure 6C are the means of the two collection timings that were pooled in each case $(28+35$ dai or $42+49$ dai). The percentage of DON in the nonbran fraction appeared to increase with increasing lateness of inoculation, especially for the susceptible P26R12, although again it was not possible to calculate confidence intervals on these values. In other words, in P26R12 in particular, earlier infections apparently led to a higher fraction of total DON in the bran than later infections.

DON/FDK ratio. The ratio of harvest-time DON concentrations to FDK for P26R12 provided some insight into which infection timings gave the highest likelihood of observing higher DON concentrations than would be predicted from the level of visible kernel damage (Fig. 7). Although significant differences were marginal, the results suggested that infections at 7 to 8 daa, rather than those at earlier or later stages, may be more likely to generate unexpectedly high levels of DON.

\section{Discussion}

The study set out to illuminate the window of susceptibility to FHB in wheat. Although every year is different, our 4 years of results do support the hypothesis that normally the period when infections lead to damaging severity, given conducive environmental conditions, is approximately a week starting at midanthesis (Zadoks GS 65). However, the actual period of susceptibility clearly depends on several factors.

One likely factor is cultivar genetics: the window of vulnerability to damaging infection was wider for the susceptible cultivar P26R12 than for the moderately resistant cultivar NC-Neuse in every year except 2012, which will be discussed below. With a disease that produces quantitative damage such as lost yield and test weight and mycotoxin contamination, susceptibility and resistance are relative terms. Susceptibility must be defined by means of certain damage parameters: for example, the ceiling of $2 \mu \mathrm{g} / \mathrm{g}$ of DON in harvested grain. In two of the four years, P26R12 was susceptible to infections resulting in DON greater than $2 \mu \mathrm{g} / \mathrm{g}$ as late as 7 to 8 daa, but not later, whereas in two other years it was susceptible by that definition as late as 13 daa (although in 2011, only at 0 and 13 daa). For the moderately resistant cultivar NC-Neuse, excessive DON concentrations were greater than $2 \mu \mathrm{g} / \mathrm{g}$ only from 0-daa infections in 2010

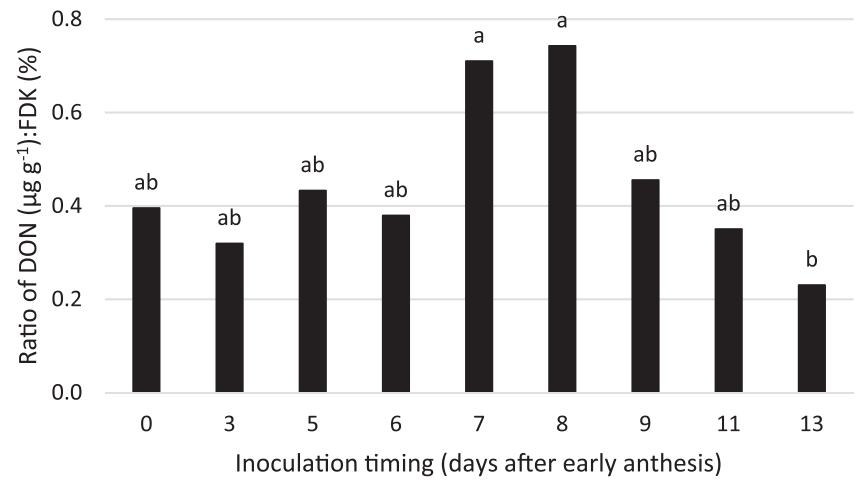

Fig. 7. Ratio of deoxynivalenol (DON) concentration to visible kernel damage, represented by Fusarium-damaged kernels (FDK), for susceptible winter wheat cultivar P26R12 inoculated with Fusarium graminearum conidia at various timings after early anthesis. Values are 4-year averages (2010 to 2013), and bars topped by the same letter are not significantly different $(P \leq 0.05)$ using least squares means. 
and 2011, and no later than 5 daa in 2013, but from all inoculation timings including 13 daa in 2012. To generalize about the differences between susceptible and moderately resistant cultivars with regard to the window of vulnerability, we would need to examine a larger set of cultivars in both classes. However, we hypothesize on the basis of the present results that one of the traits that comprise cultivar resistance is a shortened period of vulnerability to the infections that result in problematic levels of DON and kernel damage.

If indeed cultivars labeled susceptible due to their phenotype at harvest are also vulnerable to damaging infections for longer periods of time, this trait of prolonged vulnerability following anthesis should be taken into account both in breeding and in risk management. For example, in a year when FHB risk is low at the growth stages of heading and early anthesis but increases at mid- or late anthesis, the degree of cultivar susceptibility may be an important factor to consider when weighing whether to make a "late" fungicide application. That is, an application as much as a week after midanthesis, should that be the earliest possible timing, will likely be of greater urgency on a susceptible cultivar than on a moderately resistant one. In a multilocation study of winter wheat resistance combined with fungicide applications at timings ranging from $50 \%$ early anthesis to 6 to 7 days after that, the later applications tended to provide greater DON control than application at 50\% early anthesis for both moderately resistant and susceptible cultivars, although differences were not significant (Paul et al. 2019). The range of timings tested was not as wide as the window of susceptibility considered in the present study.

The extended window of susceptibility that appeared in 2012 was likely related to the impact of cold snaps on both host and pathogen. These cold snaps occurred on 2 and 3 daa, when the temperature fell below $4.0^{\circ} \mathrm{C}$ for 3 and $4 \mathrm{~h}$ each night, respectively, and again at 14 daa, when the temperature fell below $5.0^{\circ} \mathrm{C}$ for $6 \mathrm{~h}$. Anthesis is the most freeze-sensitive growth stage in wheat, and at anthesis, even temperatures somewhat above $0^{\circ} \mathrm{C}$ may cause injury with sufficiently long exposure times (Shroyer et al. 1995). We hypothesize that the cold temperatures, which approached the level and duration that can injure anthers, extended the period of host susceptibility by slowing and spreading out the flowering process, and creating more diversity of flowering stage within each plot. This is supported by comparing the growth stages in 2012 with those in the previous 2 years (Table 2): the transition from midanthesis to early milk elapsed over approximately 7 to 9 days in 2010 and 2011 but over 13 days in 2012. So, for example, the growth stage of kernel watery ripe (Zadoks GS 71) occurred at about 6 to 7 daa in 2010 and 2011 but at about 9 daa in 2012. With the growth stages from midanthesis to early milk spread out over more days in 2012, it appears there was a longer period of vulnerability to damaging levels of infection.

Although low temperatures may have prolonged vulnerability in the host by creating physiological changes, it is also possible that they changed the infection biology of $F$. graminearum, causing it to behave during a "late" infection as it normally would during an "early" one. To our knowledge, the possibility of low temperatures during anthesis extending the period of susceptibility to FHB has not been investigated, and it warrants further study, perhaps in controlled environments. If this is indeed a predictable phenomenon, it should inform the approach to fungicide timing, again implying that later fungicide applications may have relatively more benefit in years with cold snaps during anthesis.

Mean temperatures during anthesis were also relatively low in 2013 but did not dip as low. In that year, peak susceptibility occurred with the 3-daa infections rather than the 0-daa infections. However, the interval of susceptibility in 2013 as defined by harvest DON $\leq 2 \mu \mathrm{g} / \mathrm{g}$ was similar to that in the warmer year of 2010: about 8 to 9 days. Thus, the range of temperatures during anthesis in those two years, although one was warmer than the other, led to similar windows of susceptibility.

How does the development of DON during grain-fill differ depending on when infection occurs? From the present data, it appears that certain infection timings (usually 0 to 7 daa, but in one case 9 daa) can result in epidemics that produce extremely high levels of DON (e.g., 25 to $170 \mu \mathrm{g} / \mathrm{g}$ ) at early stages of grain-fill. In all three years, there was at least one infection timing for each cultivar that stood out as the most favorable for substantially higher levels of DON. Those very high levels of DON generally declined by the end of grain-fill. In the same environment, infections that occurred a few days later resulted in DON levels that never exceeded 5 to $10 \mu \mathrm{g} / \mathrm{g}$ at the maximum.

How does infection timing affect the extent to which $F$. graminearum spreads within spikes during grain-fill? Using PIK, DNA, and DON progression data, this study shed light on the dynamics of $F$. graminearum colonization of spikes during two years, 2010 and 2012. The two years presented quite different pictures. In 2010, with a relatively narrow window of susceptibility, only the 0 - and 7-daa inoculations generated elevated levels of PIK; all later infections did not. Moreover, this greater spread within spikes only developed late in grain-fill ( 35 or 42 dai). It is likely that the $5.5-\mathrm{cm}$ rainfall on 34 daa (Fig. 1) facilitated the late spread within the head evident in both cultivars. The oldest 0 -daa infections were evidently most capable of responding to the newly moist conditions. The 7-daa infections produced a similar although lesser increase in PIK at 42 dai. PIK levels in the later-infected treatments never exceeded 5 to $10 \%$ and were not similarly responsive to the 34-daa rainfall. Although the 9-daa and later infections were established to some extent inside fungal kernels, the fungus was not able to take advantage of moisture to resume spreading to new kernels. The earlier infections allowed the fungus to continue developing and multiplying even after host senescence began, whereas the later infections restricted the maximum extent of spike infection to a lower level. As expected, DNA concentrations told a similar story: in both cultivars, the 0-daa infections led to a greater amount of spread than did 7-daa infections. The relatively high level of fungal DNA in P26R12 at 42 dai reflects a late-season surge of growth that may have been precipitated by heavy rain at 34 daa.

In 2012, the year with the extended period of susceptibility due to cold snaps during anthesis, PIK and DNA concentrations were much higher than in 2010 in both cultivars. PIK increased readily following inoculations at all timings (except 0 daa, due to irrigation failure), as $F$. graminearum spread within spikes, with a tendency for earlier infection timings to continue increasing and later timings to level off in late May. There were surprisingly few differences in PIK among the infection timings, although the 9-daa infections in P26R12 tended to have higher PIK, and the 13-daa infections in NC-Neuse had lower levels. Indeed, in late May the PIK from the 3- and 9-daa infections became equal for both cultivars, reflecting the fact that the index and FDK values for those dates were relatively high, especially for P26R12. However, in P26R12, DON from the 3-daa infections was lower than DON from the 9-daa infections, both during grainfill and at harvest. This may have been because 3 daa was during the first cold snap, getting those infections off to a slow start, whereas 9 daa was between the cold snaps. Overall, similar to 2010, there was more fungal DNA from the 3-daa than from the 13-daa infections in 2012. Reflecting the extended window of vulnerability, substantially more fungal spread occurred following later (13-daa) inoculations in both cultivars than had occurred following 7-daa inoculations in 2010.

In 2012, the year of prolonged susceptibility, the differences between NC-Neuse and P26R12 in index, DON, PIK, and FDK were smaller than in other years. However, although final PIK levels were 50 to $80 \%$ for both cultivars, fungal DNA concentrations were lower in NC-Neuse than in P26R12 throughout grain-fill and at harvest from both early and late infections. Final DON and fungal DNA concentrations were higher in P26R12 than in NC-Neuse for the 8- to 13daa inoculations, indicating the relative resistance level of the two cultivars was better illustrated for the later infections. It is not clear why the unusual conditions in 2012 reduced the differential in overall resistance between the two cultivars; possibly the cold temperatures during anthesis were a factor.

We report the DON data from samples separated into bran and nonbran fractions because they suggest an interesting hypothesis regarding infection timing, which we will discuss below. First, however, our finding that the majority of the DON was in the bran agrees with most other reports, even though we were unable to utilize replication. Although the range of DON reduction from debranning 
is wide (Cheli et al. 2013), most studies of the distribution of DON in milling fractions have found the majority of the DON in the bran. For example, Nishio et al. (2010) found that the DON concentration in naturally contaminated whole spring wheat kernels was roughly doubled in the bran and halved in the flour across a wide range of total DON levels. Zhang and Wang (2014) found that an average of about $35 \%$ of whole-kernel DON was in the bran, $27 \%$ in shorts, and $9 \%$ in flour. Schwake-Anduschus et al. (2015) found that naturally contaminated wheat samples had about twice the toxin level of DON and DON-3-glucoside compared with whole grain. The fact that the majority of the DON is usually found in the bran is thought to be because the pericarp and testa, which together comprise the bran, are the first tissues to be colonized by Fusarium (Zhang and Wang 2014).

Our results suggest, although do not demonstrate, that later-infected plots had a higher nonbran DON percentage than earlier-infected plots. Also, the moderately resistant cultivar NC-Neuse generally had a higher flour DON percentage than the susceptible cultivar P26R12. Because they are not conclusive owing to the lack of repetition in the treatment combinations, both in the number of cultivars in each resistance category and in the combination of other factors, these observations need to be further tested. Additionally, the data suggest that in P26R12 under our experimental conditions, which included mist irrigation, a higher risk of low-FDK, highDON grain resulted from infections toward the end of the normal period of susceptibility (7 to 8 daa) and not from grain with very late infections at 11 or 13 daa.

These data are of interest because earlier work suggested that late infections could result in plump asymptomatic kernels with unexpectedly high DON levels (Cowger and Arellano 2010). It has also been observed that although milling to remove bran is normally expected to reduce DON by about half (e.g., from 2 to $1 \mu \mathrm{g} / \mathrm{g}$ ) in the finished white flour, on occasion the DON remaining in white flour is unexpectedly high. It is possible that grain from late infections and/or from moderately resistant cultivars generally has a higher percentage of the DON in the endosperm and germ. It is unclear why later infections should have relatively more DON in the endosperm and germ. DON is hydrophilic, and possibly the DON produced from earlier infections had more time to be transported to the interior of kernels. If this proved true after further investigation, it could be useful in guiding grain users' strategies in high-FHB years.

In conclusion, damaging infections were generally caused by infections within about 7 days of midanthesis (Zadoks GS 65), although the moderately resistant cultivar NC-Neuse had at least a 2- to 3-day shorter window of susceptibility to economically damaging infections than the susceptible cultivar P26R12. In three of the four years, only the early infections (0 to 3 days after Zadoks GS 65) allowed extremely high DON levels to develop early in grain-fill, and although those levels declined during grain-fill, they remained unacceptably high at physiological maturity. The data suggest that although infections may occur from later-arriving spores, it is usually these early infections that give rise to a high degree of fungal spread within the spike, and accompanying high levels of DON and kernel colonization and damage. By contrast, slightly later infections (7 to 8 days after midanthesis) can result in higher levels of DON than visible kernel damage might predict. Cold temperatures during anthesis may extend the period of susceptibility, possibly by drawing out the stage at which the wheat crop is most vulnerable.

\section{Acknowledgments}

We appreciate the excellent technical support of Prasad Damecharla, Matthew Hargrove, and Jennifer Patton Özkurt, and the assistance of William Berzonsky.

\section{Literature Cited}

Beccari, G., Arellano, C., Covarelli, L., Tini, F., Sulyok, M., and Cowger, C. 2019. Effect of wheat infection timing on Fusarium head blight causal agents and secondary metabolites in grain. Int. J. Food Microbiol. 290:214-225.
Brandfass, C., and Karlovsky, P. 2008. Upscaled CTAB-based DNA extraction and real-time PCR assays for Fusarium culmorum and F. graminearum DNA in plant material with reduced sampling error. Int. J. Mol. Sci. 9:2306-2321.

Cheli, F., Pinotti, L., Rossi, L., and Dell'Orto, V. 2013. Effect of milling procedures on mycotoxin distribution in wheat fractions: A review. LWTFood. Sci. Technol. 54:307-314.

Covarelli, L., Beccari, G., Prodi, A., Generotti, S., Etruschi, F., Juan, C., Ferrer, E., and Mañes, J. 2015. Fusarium species, chemotype characterisation and trichothecene contamination of durum and soft wheat in an area of central Italy. J. Sci. Food Agric. 95:540-551.

Cowger, C., and Arellano, C. 2010. Plump kernels with high deoxynivalenol linked to late Gibberella zeae infection and marginal disease conditions in winter wheat. Phytopathology 100:719-728.

Cowger, C., and Arellano, C. 2013. Fusarium graminearum infection and deoxynivalenol concentrations during development of wheat spikes. Phytopathology 103:460-471.

Cowger, C., Patton-Ozkurt, J., Brown-Guedira, G., and Perugini, L. 2009. Postanthesis moisture increased Fusarium head blight and deoxynivalenol levels in North Carolina winter wheat field experiment. Phytopathology 99:320-327.

Cowger, C., Ward, T., Nilsson, K., Arellano, C., McCormick, S. P., and Busman M. 2020. Regional and field-specific differences in Fusarium species and mycotoxins associated with blighted North Carolina wheat. Int. J. Food Microbiol. 323:108594.

Del Ponte, E. M., Fernandes, J. M. C., and Bergstrom, G. C. 2007. Influence of growth stage on Fusarium head blight and deoxynivalenol production in wheat. J. Phytopathol. 155:577-581.

European Union (EU). 2006. Commission Regulation (EC) No. 1881/2006 of 19 December setting maximum levels for certain contaminants in foodstuffs. Off. J. Eur. Union L364:5-24.

European Union (EU). 2007. Commission Regulation (EC) No. 1126/2007 of 28 September amending Regulation (EC) No. 1881/2006 setting maximum levels for certain contaminants in foodstuffs as regards Fusarium toxins in maize and maize products. Off. J. Eur. Union L225:14-17.

Hart, L. P., Pestka, J. J., and Liu, M. T. 1984. Effect of kernel development and wet periods on production of deoxynivalenol in wheat infected with Gibberella zeae. Phytopathology 74:1415-1418.

National Festival of Breads. 2020. A kernel of wheat. http://nationalfestivalofbreads. com/nutrition-education/a-kernel-of-wheat. Accessed March 11, 2020. Kansas Wheat Commission, Manhattan, KS.

Nicolaisen, M., Supronienè, S., Nielsen, L. K., Lazzaro, I., Spliid, N. H., and Justesen, A. F. 2009. Real-time PCR for quantification of eleven individual Fusarium species in cereals. J. Microbiol. Methods 76:234-240.

Nishio, Z., Takata, K., Ito, M., Tanio, M., Tabiki, T., Yamauchi, H., and Ban, T. 2010. Deoxynivalenol distribution in flour and bran of spring wheat lines with different levels of Fusarium head blight resistance. Plant Dis. 94: 335-338.

Parry, D. W., Jenkinson, P., and McLeod, L. 1995. Fusarium ear blight (scab) in small grain cereals-A review. Plant Pathol. 44:207-238.

Parry, D. W., and Nicholson, P. 1996. Development of a PCR assay to detect Fusarium poae in wheat. Plant Pathol. 45:383-391.

Pate, M. H., Sullins, R. D., and Faga, B. A. 2003. Impact of increased DON levels on the milling and baking industry. Page 1 in: 2003 National Fusarium Head Blight Forum Proceedings. S. M. Canty, J. Lewis, L. Siler, and R. W. Ward, eds. Michigan State University, East Lansing, MI.

Paul, P. A., Salgado, J. D., Bergstrom, G., Bradley, C. A., Byamukama, E., Byrne, A. M., Chapara, V., Cummings, J. A., Chilvers, M. I., Dill-Macky, R., Friskop, A., Kleczewski, N., Madden, L. V., Nagelkirk, M., Stevens, J., Smith, M., Wegulo, S. N., Wise, K., and Yabwalo, D. 2019. Integrated effects of genetic resistance and prothioconazole + tebuconazole application timing on Fusarium head blight in wheat. Plant Dis. 103:223-237.

Schmale, D. G., III, and Bergstrom, G. C. 2003. Fusarium head blight. Plant Health Instr. https://doi.org/10.1094/PHI-I-2003-0612-01

Schwake-Anduschus, C., Proske, M., Sciurba, E., Muenzing, K., Koch, M., and Maul, R. 2015. Distribution of deoxynivalenol, zearalenone, and their respective modified analogues in milling fractions of naturally contaminated wheat grains. World Mycotoxin J. 8:433-443.

Shroyer, J. P., Mikesell, M. E., and Paulsen, G. M. 1995. Spring Freeze Injury to Kansas Wheat. Report No. C-646. Kansas State University, Manhattan, KS.

U.S. Food and Drug Administration. 2010. Guidance for industry and FDA: Advisory levels for deoxynivalenol (DON) in finished wheat products for human consumption and grains and grain by-products used for animal feed. https://www.fda.gov/regulatory-information/search-fda-guidance-documents/ guidance-industry-and-fda-advisory-levels-deoxynivalenol-don-finished-wheatproducts-human

Zhang, H., and Wang, B. 2014. Fate of deoxynivalenol and deoxynivalenol-3glucoside during wheat milling and Chinese steamed bread processing. Food Control 44:86-91. 